\title{
Interferometry on Stars at Mid-Infrared Wavelengths
}

\author{
K. Tatebe ${ }^{a}$ D. D. S. Hale ${ }^{a}$ and C. H. Townes ${ }^{a}$ \\ ${ }^{a}$ Space Sciences Laboratory \\ University of California, Berkeley \\ Berkeley, CA 94720-7450
}

\begin{abstract}
The ISI (Infrared Stellar Interferometer), an interferometer operating at mid-infrared wavelengths will be discussed, including both the instrumentation and results of stellar measurements. The ISI operates in the 10 micron wavelength region using three movable telescopes and heterodyne detection with $\mathrm{CO}_{2}$ laser local oscillators. Phase closure allows rather complete imaging of stars and their dust shells, including measurement of asymmetries. Measurements have been conducted of the emission of material from stars, some ejected at very high velocity, the size and change of size of some stars, stellar ellipticity, and stellar asymmetry. Material blown off from stars is often emitted in periodic shells, and frequently asymmetric.
\end{abstract}

Keywords: infrared, interferometry, stars, dust, heterodyne

\section{INTRODUCTION}

The UC Berkeley ISI (Infrared Stellar Interferometer) is an interferometer using heterodyne receivers located at Mount Wilson Observatory operating at mid-infrared wavelengths $(9-12 \mu \mathrm{m})$. Since 2004 it has has consisted of three telescopes, an increase from the original two. Each receiver in the array is a Pfund-type telescope housed within a movable semi-trailer. The Pfund configuration gives the stability necessary for interferometric work while allowing the telescopes to be moved to a variety of locations around the site. This provides flexibility in the choice of array configurations.

Each receiver mixes starlight with a local $\mathrm{CO}_{2}$ laser oscillator onto a square-law, i.e., intensity, detector. The resulting beat frequency, the heterodyned signal, is then transmitted via coaxial cable to a central control room where appropriate delays are added and the signals correlated. The local oscillators in each telescope are phase locked to a master laser housed in a separate building. As a result the phase information of the starlight is preserved in the heterodyned signal enabling fringes to be formed. The bandwidth of this heterodyned signal, and ultimately the sensitivity of the system, is limited by the response of the detector to $\pm 2.6 \mathrm{GHz}$. A detailed description of the beam-path and optics of the telescopes has been previously published. ${ }^{1}$

With the recent addition of a third telescope it is now possible to measure three interference fringes simultaneously, and hence, closure phase. Closure phase, the sum of the phases of all three baselines, is of importance because it is not significantly affected by atmospheric phase shifts and provides a useful measure of asymmetry of the source where more intensity is found on one side of an object than the other. The first successful use of all three telescopes to measure closure phase was accomplished in $2004 .^{2}$

During its operation the ISI has measured over a dozen stars with a variety of array configurations. Most of the single baseline data were taken with the telescopes placed in an east-west orientation at several baseline distances. $^{3}$ The first three-telescope array was configured in a linear east-west configuration with baselines of 4, 8 and $12 \mathrm{~m}$. At these baselines measurements were made of the structure of the dust shells that commonly surround late-type stars. ${ }^{4}$ Recently, the telescopes have been moved into a triangular, or Y shaped, array with baselines of approximately $35-40 \mathrm{~m}$. This array configuration was chosen in order to optimize measurements of asymmetries of the stars themselves, separated from surrounding dust and gas.

Further author information: (Send correspondence to K. T)

K. T.: E-mail: tatebe@ssl.berkeley.edu, Telephone: 5106429500

Infrared Spaceborne Remote Sensing and Instrumentation XV, edited by Marija Strojnik-Scholl, Proc. of SPIE Vol. 6678, 66781G, (2007) · 0277-786X/07/\$18 - doi: 10.1117/12.734328 


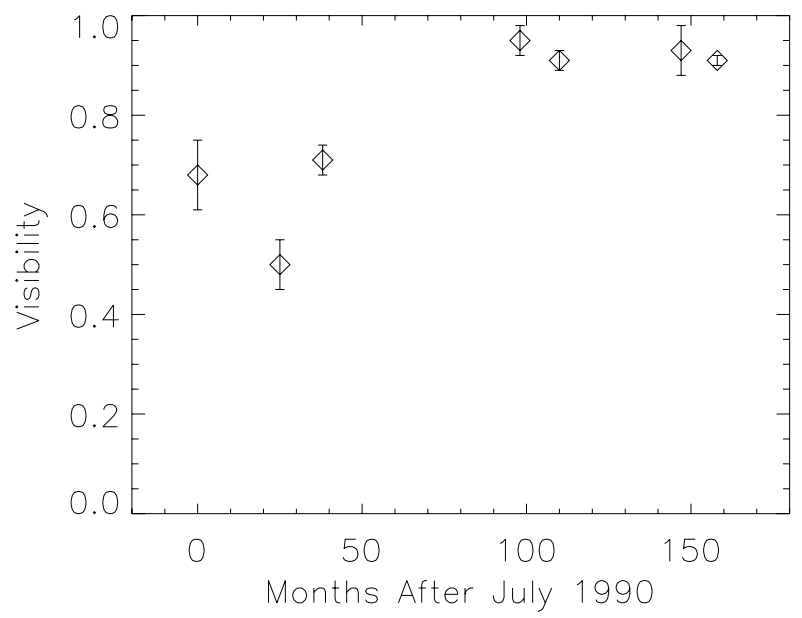

Figure 1. Visibilities of $\alpha$ Her, spanning over a decade, at an average spatial frequency of $7 \mathrm{SFU}\left(7 \times 10^{5} \mathrm{cycles}^{\mathrm{rad}}{ }^{-1}\right.$.

\section{SCIENTIFIC OBJECTIVES}

The ISI operates at wavelengths well-suited to the study of cooler stars or warm dust, which glow brightly in this region of the spectrum. This makes late-type stars ideal objects to study as they are characterized by low surface temperatures, large sizes and sometimes by periodic variations in brightness. These stars are often expanded to two orders of magnitude larger than the Sun, and are often surrounded by gas and dust. Because of their size they have low surface gravity, thus reducing the forces holding the star in a spherical shape. Further, the complex interactions within the star result in regular pulsations for some of them that change the brightness and size of the star. The resulting flows of gas can create turbulence, shocks and can serve to eject a shell of material from the surface of the star. These forces may, with relatively low surface gravity to hold them in check, significantly distort the surface of the star from the normal spherical symmetry, and may also result in the non-uniform outflow of ejected material.

\section{SINGLE BASELINE MEASUREMENTS}

Visibility measurements taken using a single baseline provide information about the size of a dust shell surrounding a star. The long history of measurements at the ISI allows for the tracking and comparison of the sizes of these dust shells over time. Data from one star in particular, $\alpha$ Her, are striking and indicate that material may have been ejected at more than three times the velocity typical for late-type stars. ${ }^{5}$ These observations, spanning the last two decades, are displayed in Figure 1 and show a rapid fall and subsequent rise in visibility at spatial frequencies where the dust is thought to be nearly fully resolved while the star is almost completely unresolved. At these intermediate baselines, with spatial frequencies in the range of 6-12 SFU, the star should be unresolved, thus yielding a visibility near unity if no dust is present. SFU stands for "Spatial Frequency Unit" and is defined to be $10^{5}$ cycles $\mathrm{rad}^{-1}$. Once a shell of dust is emitted, the large size of the dust shell will result in the visibility decreasing as the shell is resolved by the interferometer. As the dust further expands, it will cool and dissipate thus no longer emitting significant radiation in the mid-infrared leaving only the star visible; this will result in the visibility climbing again to near unity.

Hence, a simple interpretation of the visibilities shown in Figure 1 indicates an emission of material in 1989 which subsequently expanded rapidly into a dust shell contributing approximately half the total light of the object in the mid-infrared. The subsequently rapid rise in the visibility suggests the dust then dissipated just as quickly, leaving only the star visible by sometime around late 1996.

While the interpretation of these data as the rapid outflow of a dust shell is straightforward, the actual speed required of the dust to cause such rapid changes in the visibility is likely to be at least $72 \mathrm{~km} \mathrm{~s}^{-1}$. This is in 
contrast to typical outflow velocities of $25 \mathrm{~km} \mathrm{~s}^{-1}$ or less. This is difficult to account for as the velocity of the dust is primarily due to acceleration from radiation pressure, and does not vary strongly enough with stellar luminosity to make excess luminosity a likely explanation for the high velocity observed. It is possible that a very high dust to gas ratio, thus minimizing viscous drag effects, could account for the high velocity observed, though there is no reason to expect this. Alternatively, a different acceleration mechanism may be responsible.

\section{DUST SHELL IMAGING}

From 2004 until 2006, with the telescopes in a linear array, information on stars and the structure of the dust surrounding them was measured along one direction. Spatial frequency information derived from 4,8 and $12 \mathrm{~m}$ baselines, all in the east-west direction, is appropriate for determining the one-dimensional profiles of the dust shells surrounding these stars. A full image of the dust shell, even along one direction, requires the recovery of visibility phase information from the closure phase data.

For a linear array, if the closure phase is plotted as a function of the spatial frequency of the shortest baseline it can usually be fitted well by a polynomial function, $\Phi_{c p}(x)$, and in these cases the visibility phase as a function of spatial frequency, $\phi(x)$ can be determined. So long as the object has no sharp structure in the dust, as is typically the case, a polynomial usually fits the phase closure data rather well. For a real image the phase should be an odd function, thus only odd terms should be non-zero. Also, only a few polynomial terms are used since there are a finite number of points. The phase and closure phase can be represented as sums, that are functions of spatial frequency and the spatial frequency of the shortest baseline, respectively:

$$
\phi(x)=\sum_{n=3}^{n_{\max }} a_{n} x^{n} \quad \Phi_{c p}(x)=\sum_{m=3}^{m_{\max }} b_{m} x^{m} .
$$

In this case the closure phase is then given as the sum of the three individual baseline phases.

$$
\Phi_{c p}(x)=\phi(x)+\phi(2 x)-\phi(3 x)
$$

If such a smooth polynomial curve can be satisfactorily fit to the closure phase then Equation 2 can be solved, after substituting the series expressions for $\phi(x)$ and $\Phi_{c p}(x)$, to give a polynomial function representing the visibility phase, $\phi(x)$, as a function of spatial frequency: ${ }^{4}$

$$
\phi(x)=\sum_{n=3}^{n_{\max }} \frac{b_{n} x^{n}}{1+2^{n}-3^{n}}
$$

where $b_{n}$ are the polynomial coefficients fit to the closure phase. The constants 1,2 and 3 are specific to the these baselines which are in a ratio of $1: 2: 3$.

In general, there are not enough data to make a one-dimensional image without making some minor prior assumptions. As stated, the closure phase must be a smooth, analytic function. Also, the visibility values at spatial frequencies higher and lower than those measured must be assumed. At low frequencies the data are smoothly connected to unity visibility at zero spatial frequency. The high spatial frequency is usually assumed to be due to the star if the dust is thin. In these cases the visibility curve of a uniform disk (UD) is assumed for spatial frequencies higher than those measured, in which case the closure phase is zero. If the dust is known to be thick, the visibility is tapered gradually to zero.

This procedure has been used in the one-dimensional imaging of a number of stars. One star for which the ISI has taken a large amount of data is Mira. ${ }^{6}$ To date, the dust surrounding Mira has been measured during four separate epochs at these baselines. The images resulting from the processing of these data are shown, stacked, in Figure 2. The top-most profile shows a clear shell of dust close in to the star with a peak intensity at about 65 mas from the center of the star. The innermost peak of intensity in the dust moves out at a fairly constant

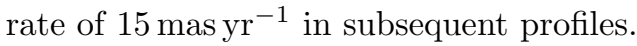

Proc. of SPIE Vol. 6678 66781G-3 


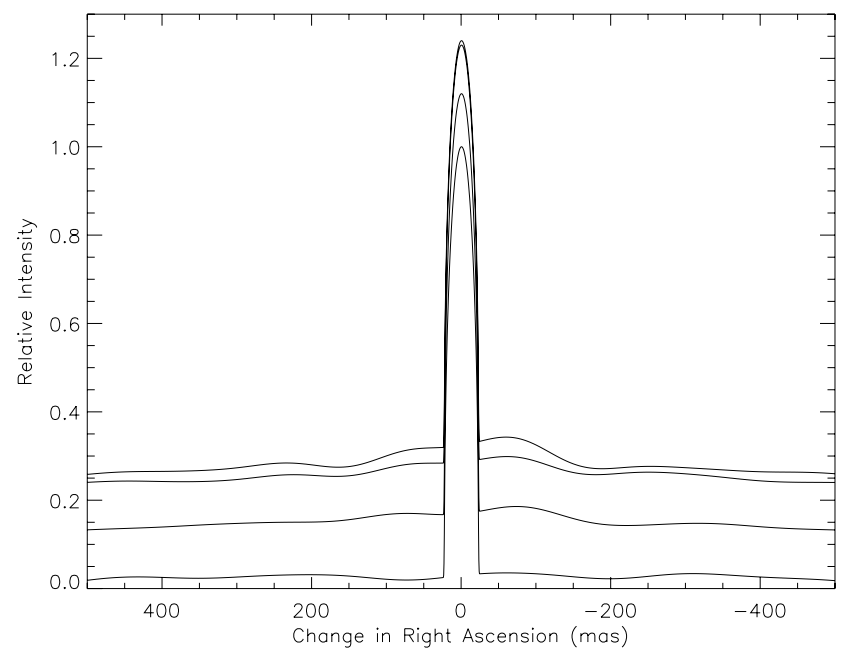

Figure 2. One-dimensional profiles of the dust shell surrounding O Ceti over four epochs during Aug. and Sept. in 2003, Oct. and Nov. of 2003 and late 2004 and 2005. The profiles are in decending order with the vertical displacement of each image from zero being proportional to time before the last epoch. Each profile is adjusted so that the maximum intensity is equal to unity before displacement. East is left.

\section{TRIANGULAR BASELINES}

In the linear east-west configuration there was sufficient resolution to measure the size and structure of the dust shells that surround many of these stars, but not to resolve the stars themselves. To gauge the size and shape of the stars longer baselines are required. Stellar diameters have been measured at the ISI using longer baselines of up to $56 \mathrm{~m}$ when it was in its two telescope form. ${ }^{7}$ By measuring the spatial frequency of the null in visibility, the diameter, assuming the shape of a uniform disk, was determined. Often there was enough data to fit the stellar visibility curve to a UD model with a determined radius and therefore use more information than just the spatial frequency of the visibility null.

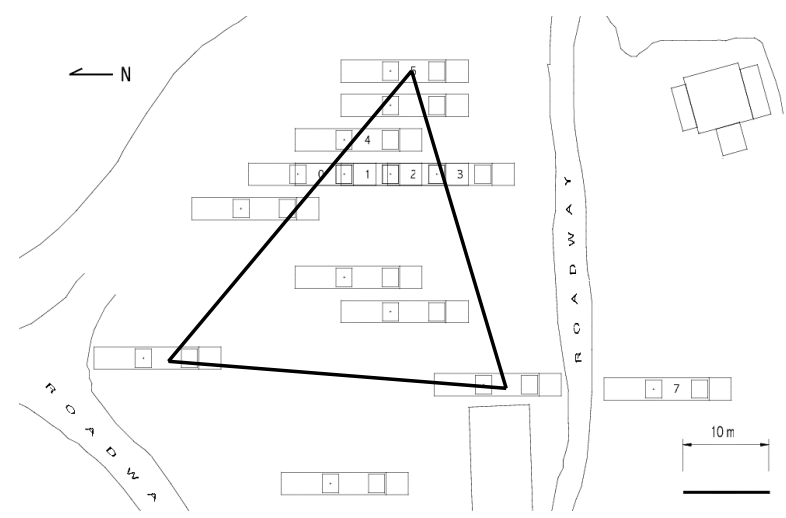

Figure 3. A schematic of the ISI site at Mount Wilson Observatory. Thick lines connect the pads where the telescopes are currently positioned. 


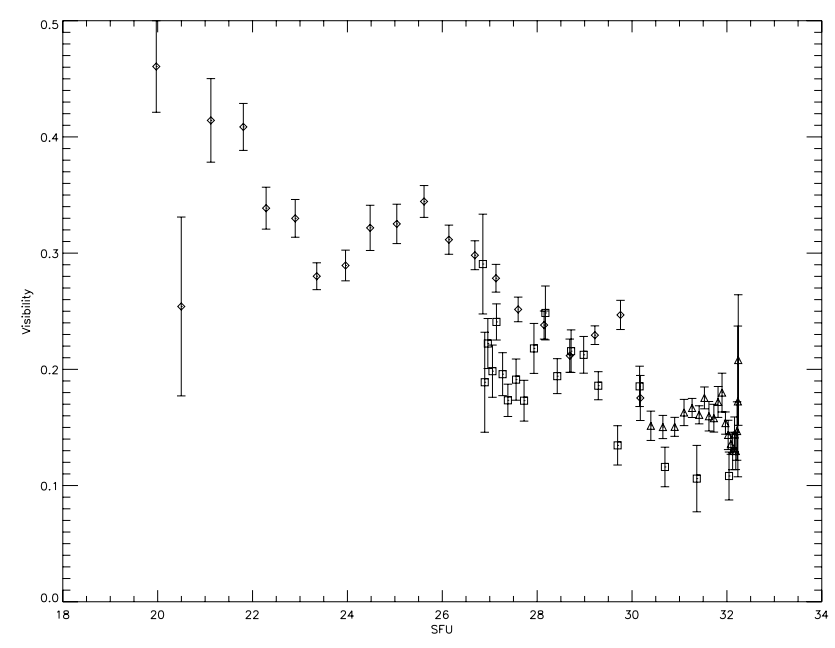

Figure 4. Visibility data for R Leo taken with the ISI in a triangular baseline configuration. Data from baseline (1-2) is plotted as diamonds, (2-3) by triangles and (3-1) by squares.

Recently, the telescopes have been moved into a triangular configuration with baselines of $\sim 40 \mathrm{~m}$. This current layout of the telescopes is shown in Figure 3. These baselines are much longer than those used to resolve the dust alone in the east-west configuration but still somewhat shorter than the long baselines used in the single baseline stellar diameter measurements. These lengths and configuration were chosen to optimize the array to detect asymmetries, i.e., deviations from circular symmetry, rather than to make the most accurate absolute size measurements. These considerations will be discussed in more detail in $\S 6$. Using such a triangular arrangement it is possible to determine the size of the star along three different directions simultaneously. A symmetric star would, of course, yield the same UD visibility curve as a function of spatial frequency for all three baselines. Any deviations from this symmetric behavior can be helpful in determining the morphology of the star. For stars near the ecliptic, such as $\alpha$ Ori, the ISI array appears as a nearly equilateral triangle during one point during the night. Measurements around this time are particularly useful as they can conclusively rule out a spherically symmetric structure for the star if the visibilities differ in spite of incomplete $u v$ coverage. This is because unequal visibilities for the three baselines at different position angles but the same spatial frequencies necessitates a departure from circular symmetry.

The use of somewhat shorter "long baselines" provide two main advantages. First, since the change in visibility with radius is largest near the steep part of the visibility curve for a UD it is advantageous to measure variations in the visibility between baselines at these spatial frequencies. This provides the most sensitive measure of changes in radius along the directions of the various baselines. Secondly, by measuring at spatial frequencies where the visibilities are not near zero it is still possible to take closure phase measurements. If any one of the three visibilities approaches too near a null, i.e., is zero to within the signal-to-noise, the phase will be dominated by noise. By keeping the signal-to-noise high, the fringe phases are much stronger than the noise, thus keeping the phase variations small and allowing accurate measurements to be made with averaging.

Since the visibility is not measured out to the null, calibration becomes an important factor in determining the size of the star. When the null is measured, there is no great concern about the proper calibration of the visibility since a visibility of zero will remain zero regardless of calibration. Calibration of visibility for each baseline is done by measuring circular stars of known size, $\alpha$ Tau and $\alpha$ Boo. These calibrations, and the assumed size of the calibrators, appear accurate as the present calibrations yield stellar diameters for Mira that are consistent with past diameters measured at the null. ${ }^{7}$ Additionally, the radius of Mira has been found to vary with stellar phase to a degree consistent with these past measurements.

The ability to measure visibilities and closure phases at spatial frequencies that resolve the star is very useful for characterizing any asymmetry in the intensity distribution of the star. Visibilities for the star R Leo are 


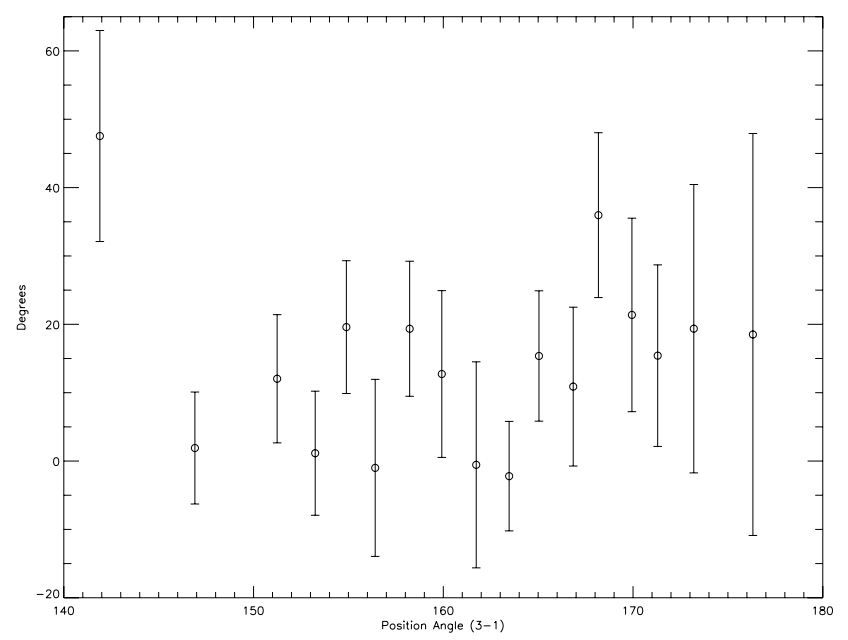

Figure 5. Closure phase data of R Leo taken with the ISI in a triangular baseline configuration. Phases are in degrees and are plotted as a function of the position angle of the (3-1) baseline.

plotted as a function of spatial frequency in Figure 4. Closure phases for R Leo are plotted in Figure 5 as a function of the position angle of the (3-1) baseline. From the the visibility curve alone it is apparent the star is not spherical as evidenced by the differing visibilities between baselines as similar frequencies. Data from each baseline is plotted as a different symbol. In addition to this non-sphericity, though, there also appears to be a significant lopsidedness to the star based on the closure phase being best fit by a curve which deviates from zero by as much as 20 degrees.

\section{DISCUSSION OF CLOSURE PHASE FOR TRIANGULAR BASELINES}

The phase of a fringe generated by a single baseline is simply interpreted as the phase of the Fourier component measured at a particular spatial frequency. The relationship between this fringe phase and closure phase was discussed somewhat in $\S 4$ for the case of a linear array in which all three baselines were aligned along a single direction. If the baselines are arranged in a triangle, as in Figure 3, the closure phase is more difficult to interpret intuitively.

In order to gain a better understanding of how closure phase behaves for a triangular array it is instructive to examine what closure phase would be measured in a simplified case. Perhaps the easiest triangular array to examine is one in which the telescopes are arrayed in the form an equilateral triangle pointing north. For a second simplification, the asymmetric star is modeled as a simple uniform disk with a point source of additional intensity located at some angle around the edge of the star. As a final simplification, the star is assumed to be directly overhead. Under these circumstances, the closure phase measured by the array can be plotted as a function of the orientation of the point source around the perimeter of the star. This is shown as a polar plot in Figure 6 where the radial distance of the curve from the origin is the amount of closure phase measured as a function of the position angle of the point source, which is given by the angle a point on the curve makes counter clockwise from the y-axis. For example, when the point source is on the top edge of the star, at an angle of zero, the measured closure phase is zero. If, on the other hand, the point source is located on the far-right edge of the star, at a position angle of $270^{\circ}$, then the closure phase is $7.63^{\circ}$. It is interesting to note that the opposite asymmetry, with the point to the left, generates the same closure phase but with opposite sign. Changes in the asymmetry of the star, i.e., as the point is moved farther or closer to the center of the star, or is made more or less intense, will change the magnitude of the measured closure phases. Thus, these changes will alter the size of the daisy-shaped plot in Figure 6, but will not affect its shape for the case of an equilateral triangle of baselines.

In general, as the star travels across the sky, the triangle of telescopes will not remain equilateral. This will change the shape of the daisy in Figure 6 by altering the size, width and number of petals as well as their 


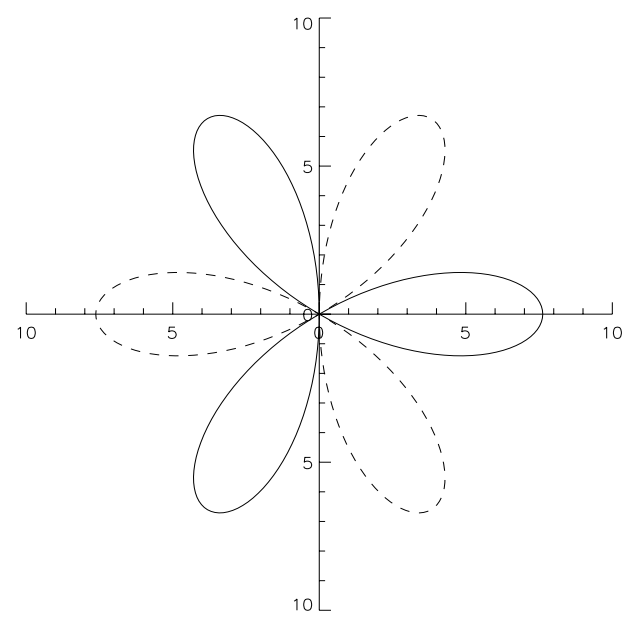

Figure 6. Polar plot of closure phase as a function of position angle for a uniform disk with point source. Thus for a given position angle of the point source, the $\theta$ coordinate, the curve indicates the measured closure phase, the $r$ coordinate. Solid curves indicate positive values while dashed curves indicate negative values.

orientation. The amount of asymmetry can also change the shape of the daisy when the baselines are not equilateral. This is significant since there are some orientations for which even a large asymmetry will result in a zero closure phase, e.g., at $0^{\circ}$. Thus, it is important to take data over a variety of projected position angles, i.e., at many times during the night, to avoid such "blind spots" where asymmetries in certain directions might not be detected.

\section{OPTIMAL BASELINE LENGTH}

The optimum spacing of telescopes in the triangular configuration is determined by finding the spatial frequency at which the visibility will change the most for changes in stellar size. Figure 7 shows the visibility curves of a 20 mas radius UD star along with those $5 \%$ and $10 \%$ bigger on the left. The plot on the right shows the relative changes in the visibility for an infinitesimal, $5 \%$ and $10 \%$ change in stellar size.

The peak of the derivative of visibility can be found by differentiating the analytical form for the visibility of a UD. The analytical expression for the visibility of a UD is relatively simple and is given by

$$
V(r, x)=\frac{J_{1}(2 \pi r x)}{\pi r x}
$$

where $x$ is the spatial frequency in $\operatorname{arcsec}^{-1}$ and $r$ is the radius in arcseconds. Maximizing the derivative by solving for the roots of the second derivative of $V$ with respect to $r$, i.e., $\partial_{r r} V(r, x)$, however, is complicated but can be readily solved using numerical methods. It is found that the spatial frequency at which the visibility changes most is given by $x=0.48 / r$. Interestingly if $x$ is measured in SFU rather than $\operatorname{arcsec}^{-1}$ the result is $x=1.00 / r$. These are equivalent to the spatial frequency that is $79.4 \%$ that of the first null. If instead, the change in visibility-squared is maximized then $x$, in cycle $\operatorname{arcsec}^{-1}$, is given by $x=0.32 / r(x=0.66 / r$ if $x$ is in SFU). This is $52.5 \%$ of the spatial frequency of the first null, about where it might be expected.

A typical radius for the stars measured at the ISI is about 0.020 arcsec giving an maximum variation in visibility at a baseline of $56 \mathrm{~m}$ or $37 \mathrm{~m}$ for the greatest change in visibility-squared. The desire for baselines of $\sim 40 \mathrm{~m}$, in conjunction with the need for the trailers to be placed on one of several concrete pads located around the site, resulted in the currently realized configuration. 

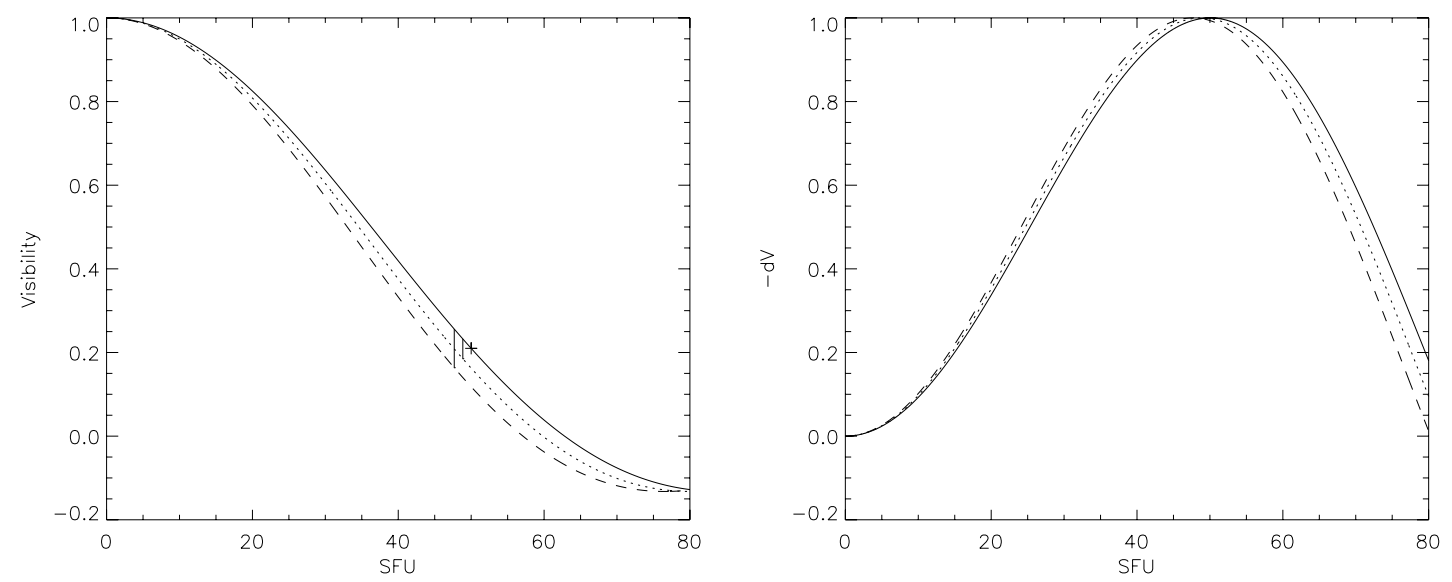

Figure 7. The left plot shows the visibility curves of a 20 mas star (solid) and those of a 21 mas (dotted) and 22 mas star (dashed). The right plot shows the negative change in visibility with an infinitesimal (solid), $5 \%$ (dotted) and $10 \%$ change is stellar radius. These curves are scaled to have a maximum height of unity. Vertical bars in the left plot show the location of maximum visibility change between the original and enlarged stars. The "+" indicates where the derivative is maximized.

\section{FUTURE DIRECTIONS}

When characterizing the size and asymmetry of giant stars it becomes important to define what is meant by the surface of the star. A well-defined photosphere, such as occurs for a smaller star like the Sun, becomes difficult to pin down in giants whose extended envelopes decrease in density very gradually with altitude. This gradual change in density results in measurements of stellar radius at different wavelengths that vary by as much as a factor of two. These variations are the result of different opacity mechanisms coming into play at different wavelengths. Currently, it is not well understood which opacity mechanisms dominate in the mid-infrared. To gain a better understanding of the opacity mechanisms it is important to separate effects due to molecular lines and those due to continua. To this end the ISI, with its heterodyne receivers, generates radio-frequency signals that can be processed electronically to form high resolution spectra. ${ }^{8}$ At these narrow bandwidths, the heterodyne detection scheme will be competitive in signal-to-noise with direct-detection systems. New high-speed digitizing electronics will make this process even more efficient thereby enabling more accurate measurements of the stellar size in spectral bands of molecular transitions and in continuum regions.

\section{SUMMARY}

The ISI has conducted observations of late-type stars using various two and three baseline configurations. The multi-epoch data, spanning two decades, for the single baseline data have provided insight into apparent outflows of material at velocities higher than are typically expected. Later data, using the three telescopes in a linear array, have been used to perform basic imaging on the dust shells surrounding several stars. In the case of Mira these one-dimensional constructions have shown stable outward motion of the dust over four epochs at outflow velocities typical of these variables. Finally, the telescopes have now been placed into a triangular configuration, optimized for the detection of stellar asymmetries. Preliminary observations of $\mathrm{R}$ Leo using this configuration have clearly indicated an asymmetry based both on variations in visibility with baseline and the non-zero closure phase.

\section{ACKNOWLEDGMENTS}

The authors would like to thank Ed Wishnow and Walt Fitelson for their helpful advice. This research has been supported by the Office of Naval Research, the National Science Foundation, and the Gordon and Betty Moore Foundation. 


\section{REFERENCES}

1. D. D. S. Hale, M. Bester, W. C. Danchi, W. Fitelson, S. Hoss, E. A. Lipman, J. D. Monnier, P. G. Tuthill, and C. H. Townes, "The Berkeley Infrared Spatial Interferometer: A Heterodyne Stellar Interferometer for the Mid-Infrared," ApJ 537, pp. 998-1012, July 2000.

2. D. D. Hale, J. Weiner, and C. H. Townes, "ISI: recent technology and science," in New Frontiers in Stellar Interferometry, Proceedings of SPIE Volume 5491. Edited by Wesley A. Traub. Bellingham, WA: The International Society for Optical Engineering, 2004., p.490, W. A. Traub, ed., Presented at the Society of Photo-Optical Instrumentation Engineers (SPIE) Conference 5491, pp. 490-+, Oct. 2004.

3. W. C. Danchi, M. Bester, C. G. Degiacomi, L. J. Greenhill, and C. H. Townes, "Characteristics of dust shells around 13 late-type stars," AJ 107, pp. 1469-1513, Apr. 1994.

4. K. Tatebe, A. A. Chandler, D. D. S. Hale, and C. H. Townes, "Characterization of Dust Shell Dynamics and Asymmetry for Six Mira-Type Stars," ApJ 652, pp. 666-680, Nov. 2006.

5. K. Tatebe, D. D. S. Hale, E. H. Wishnow, and C. H. Townes, "Observation of a Burst of High-Velocity Dust from $\alpha$ Herculis," ApJL 658, pp. L103-L106, Apr. 2007.

6. A. A. Chandler, K. Tatebe, E. H. Wishnow, D. D. S. Hale, and C. H. Townes, "Asymmetries and outflows in the circumstellar dust of mira a," ApJ accepted, 2007.

7. J. Weiner, W. C. Danchi, D. D. S. Hale, J. McMahon, C. H. Townes, J. D. Monnier, and P. G. Tuthill, "Precision Measurements of the Diameters of $\alpha$ Orionis and Ceti at 11 Microns," ApJ 544, pp. 1097-1100, Dec. 2000.

8. J. D. Monnier, W. C. Danchi, D. S. Hale, P. G. Tuthill, and C. H. Townes, "Mid-Infrared Interferometry on Spectral Lines. III. Ammonia and Silane around IRC +10216 and VY Canis Majoris," ApJ 543, pp. 868-879, Nov. 2000. 